\title{
Overview on the Developments and Applications of Hesitant Fuzzy Sets: An Uncertain Decision Making Tool
}

\author{
Bin Zhu \\ School of Economics and Management, Tsinghua University, Beijing 100084, China \\ E-mail:binzhu@263.net \\ Zeshui Xu \\ Business School, Sichuan University, Chengdu, Sichuan 610064, China \\ E-mail:xuzeshui@263.net \\ Received 18 February 2016
}

Accepted 28 April 2016

Abstract

Recently, hesitant fuzzy sets perform as an emerging tool in decision making under uncertainty. This paper reviews the main relevant papers, which are published in international academic journals from 2010 to 2016, relating to the interpretations, modellings, and applications of hesitant fuzzy sets. Moreover, some possible future research directions are put forward.

Keywords: Hesitant fuzzy set, decision making, aggregation, measure, clustering

\section{Introduction}

To deal with vague or imprecise phenomena in decision making, Zadeh (1965) developed the fuzzy sets (Z-FSs) that have wide applications in various fields (Zimmermann, 2001). As a new extension, Torra (2010a) proposed the concept of hesitant fuzzy sets (HFSs) to enhance the modelling abilities of Z-FSs. Compared with other extensions of Z-FSs, such as Atanassov's intuitionistic fuzzy sets (Atanassov, 1986) (A-IFSs), type-2 fuzzy sets (Dubois, 1980; Zadeh, 1975) (T2FSs) and fuzzy multisets (Yager, 1986) (FMSs), the HFS defines the membership degree of an element associated with several possible values. As introduced by Torra (2010a), the difficulty of establishing the membership degree is not because we have a margin of error (as in A-IFSs), or some possibility distribution (as in T2FSs) on the possible values, but because we have a set of possible values. The typical application of HFSs can arise in uncertain decision making, where the decision makers (DMs) experience hesitancy in providing their judgments.
Ever since Torra (2010a) introduced HFSs, this concept has become a hot topic and received more and more attentions recent years. Numerous relevant papers have published in international journals from 2010 to 2016. We now roughly group these papers, and then give a brief review of them.

\section{Hesitant fuzzy set and several extensions}

\subsection{Hesitant fuzzy set}

Torra (2010a) defined the concept of HFS as follows:

Definition 1 (Torra, 2010a). Let $X$ be a fixed set, then a HFS on $X$ is in terms of a function $h$ that returns a subset of $[0,1]$.

HFSs have close relationship with the existing fuzzy sets, including Z-FSs, A-IFSs, T2FSs and FMSs. Specifically, HFSs are IFSs when HFSs are nonempty closed intervals, and HFSs can be represented as FMSs or T2FSs. 
Torra (2010a) considered that a typical HFS is a finite subset of $[0,1]$. In particular, the typical HFS $h(x)$ represents the possible membership values of the set at $x$. Based on this assumption, Torra (2010a) developed some basic operations of HFSs, such as the complement, the union, the intersection and the envelope of HFSs.

To further apply HFSs to decision making under uncertainty, Xia and Xu (2011) restated the concept of HFSs and defined hesitant fuzzy elements (HFEs) which are convenient to be used to present the decision makers' (DMs) preferences in decision making.

Definition 2 (Xia and Xu, 2011). Let $X$ be a fixed set, a HFS on $X$ is in terms of a function that when applied to $X$ returns a subset of $[0,1]$, which can be represented as the following mathematical symbol:

$$
E=\left\{<x, h_{E}(x)>\mid x \in X\right\}
$$

where $h_{E}(x)$ is a set of some values in [0,1], denoting the possible membership values of the element $x \in X$ to the set $E$. For convenience, $h=h_{E}(x)$ is called a HFE.

For three HFEs $h, h_{1}$ and $h_{2}$, Xia and Xu (2011) developed some new operations:

1) $h^{\lambda}=\bigcup_{\gamma \in h}\left\{\gamma^{\lambda}\right\}$;

2) $\lambda h=\bigcup_{\gamma \in h}\left\{1-(1-\gamma)^{\lambda}\right\}$;

3) $h_{1} \oplus h_{2}=\bigcup_{\gamma_{1} \in h_{1}, \gamma_{2} \in h_{2}}\left\{\gamma_{1}+\gamma_{2}-\gamma_{1} \gamma_{2}\right\}$;

4) $h_{1} \otimes h_{2}=\bigcup_{\gamma_{1} \in h_{1}, \gamma_{2} \in h_{2}}\left\{\gamma_{1} \gamma_{2}\right\}$.

Then Zhu et al. (2012b) developed two relationships for the operations:

1) $\lambda\left(h_{1} \oplus h_{2}\right)=\lambda h_{1} \oplus \lambda h_{2}$;

2) $\left(h_{1} \otimes h_{2}\right)^{\lambda}=h_{1}^{\lambda} \otimes h_{2}^{\lambda}$.

Furthermore, Verma and Sharma (2013) systematically investigated the properties of the operations of HFEs, which is helpful for the development of HFSs. To make HFSs more important and applicable, Verma and Sharma (2013) then proposed four new operations of HFSs and studied their properties. Liao and Xu (2014b) recently developed the subtraction and division operations over HFSs, which is important in forming the integral theoretical framework of HFSs.

To compare HFEs, Xia and Xu (2011) proposed a score function defined as follows:
Definition 3 (Xia and $\mathrm{Xu}, 2011$ ). For a HFE $h$, $s(h)=1 / \# h \sum_{\gamma \in h} \gamma$ is called the score function of $h$, where $\#$ is the number of the elements in $h$. For two HFEs $h_{1}$ and $h_{2}$, if $s\left(h_{1}\right)>s\left(h_{2}\right)$, then $h_{1}>h_{2}$; if $s\left(h_{1}\right)=s\left(h_{2}\right)$, then $h_{1}=h_{2}$.

Based on the assumption that two HFEs have the same number of elements and the elements are arranged in an increasing order, Farhadinia (2013b) then developed a new score function that meets some properties shown in Definition 4.

Definition 4 (Farhadinia, 2013b). Let $h=\bigcup_{\gamma \in h}\{\gamma\}=\left\{\gamma_{j}\right\}_{j=1}^{\# h}$ be a HFE, where $\# h$ returns the number of values in $h$. A score function $s$ of a HFE $h$ is defined by

$$
s(h)=\frac{\sum_{j=1}^{\# h} j \gamma_{j}}{\sum_{j=1}^{\# h} j}=\frac{2}{\# h(\# h+1)} \sum_{j=1}^{\# h} j \gamma_{j}
$$

The score functions are used to aggregate the elements in the HFE. Xia and Xu (2011)'s score function pays attention to the overall arguments fairly, whereas Farhadinia (2013b)'s score function assigns more weights to the arguments whose values are high For example, for two HFEs $h_{1}=\{0.2,0.3,0.7\}$ and $h_{2}=\{0.1,0.4,0.7\}$, according to Definition 3, we have $s\left(h_{1}\right)=s\left(h_{2}\right)=0.4$, so $h_{1}=h_{2}$. However, according to Definition 4 , we get $s\left(h_{1}\right)=0.48$ and $s\left(h_{2}\right)=0.5$, such that $h_{1}<h_{2}$.

On the other hand, the ordered weighted averaging (OWA) aggregation operators (Yager, 1988) can also be applied to aggregate the elements in the HFE. In addition, the score functions are special cases of the OWA aggregation operators. With different OWA aggregation operators (Yager, 1993), HFEs can be compared relying on different decision environment.

\subsection{Dual hesitant fuzzy set}

Zhu et al. (2012a) considered the situation where there are several possible values both for the membership degree and the non-membership degree, then defined the dual hesitant fuzzy set (DHFS) in terms of two functions that return two sets of possible values indicating the membership degree and non-membership degree respectively for each element in the domain. As a more comprehensive fuzzy set, DHFSs can encompass 
A-FSs, A-IFSs, HFSs, and FMSs as special cases with specific conditions.

To apply DHFSs to decision making under uncertainty, dual hesitant fuzzy elements (DHFEs) (Zhu et al., 2012a) were developed to present the DMs' preferences. HFFEs are more comprehensive than HFEs due to its consideration of both membership and nonmembership. For example, if 0.1 and 0.2 are considered as the possible values for the membership degree, 0.3 , 0.4 and 0.5 are considered as the possible values for the non-membership degree, then a DHFE indicating such a case can be denoted by $d=\{\{0.1,0.2\},\{0.3,0.4,0.5\}\}$. Furthermore, to develop basic operations and aggregation operations of DHFSs, Zhu et al. (2012a) developed an extension principle based on the ordered modular average (OMA) (Mesiar and MesiarováZemánková, 2011). However, since DHFSs can reduce to some existing fuzzy sets with specific conditions, $\mathrm{Zhu}$ and $\mathrm{Xu}$ (2014) further defined the typical DHFS (T-DHF) to distinguish DHFSs from A-FSs, A-IFSs and HFSs. They also defined typical DHFEs (T-DHFEs) so as to apply T-DHFSs to decision making. Basic operations and their properties of T-DHFEs were studied extensively.

Due to the advantages of DHFSs in presenting hesitant fuzzy information, Chen et al. (2014) developed the correlation and correlation coefficient of DHFSs, then investigated the pattern recognition problems about the classification of metal materials based on dual hesitant fuzzy information. Ye (2013) proposed a new correlation coefficient between DHFSs as a new extension of the existing correlation coefficients. Wang et al. (2015) developed some dual hesitant fuzzy aggregation operators, and then used them to multicriteria decision making problems.

\subsection{Extended hesitant fuzzy set (EHFS)}

Torra (2010b) gave an example to show the application of HFSs in decision making: two DMs discuss the membership degree of $x$ into $A$, one wants to assign 0.5 and the other 0.6 , which can be denoted by a HFE, $h=\{0.5,0.6\}$. However, if the two DMs both assign the value 0.5 , we can only save one value in the $h$ and loss the other one, which appears to be an information loss problem of HFSs. Since the DMs often have different importances in decision making, the loss of information provided by the important DMs may lead to ineffective results. Moreover, the preferences in $h$ can not be distinguished to the two DMs, respectively. Thus HFEs are often used to present preferences provided by the anonymous DMs whose weights are the same. Clearly, different DMs can not be identified in such case. A similar assumption, that is the DMs providing their preferences anonymous, is also in $\mathrm{Xu}$ and Xia (2011b) in developing distance measures of HFSs. In addtion, DHFSs also have this problem.

To overcome the information loss problem and identify different DMs in decision making, $\mathrm{Zhu}$ and $\mathrm{Xu}$ (2016) proposed the concept of extended hesitant fuzzy set (EHFS), where the values provided by the DMs are collected by several possible value-groups. For example, if one DM assigns 0.5 , another assigns 0.5 or 0.6 to the membership degree of $x$ into $A$, then two possible value-groups are $(0.5,0.5)$ and $(0.5,0.6)$, where the first component of the value-group is provided by the first DM, and the second provided the other. In such a case, all the preferences provided by the DMs are saved and distinguished clearly.

Zhu and $\mathrm{Xu}$ (2016) further developed extended hesitant fuzzy elements (EHFEs), and some basic operations of EHFEs. So the case mentioned above can be denoted by an EHFE as $H=\{(0.5,0.5),(0.5,0.6)\}$. Obviously, EHFSs are defined as the Cartesian product of HFSs. This definition implies that HFSs can be used to construct EHFSs. On the contrary, EHFSs can also reduce to HFSs. As a special case of EHFSs, all the operations of HFSs are consistent with the operations of EHFSs.

Zhu and Xu (2016) developed a concept of reduced EHFEs to investigate the relationship between HFSs and EHFSs. The relationships among EHFSs, A-IFSs, EHFSs and DHFS have been studied. To show the advantages of EHFSs in overcoming the drawbacks of HFSs, Zhu and Xu (2016) developed some distance measures of EHFEs, and then gave a comparative example in dealing with a multi-criteria decision making problem. These results show that the existing hesitant distance measures can be considered as a particular case of the extended hesitant distance measures.

\subsection{Interval-valued hesitant fuzzy set and generalized hesitant fuzzy set}

Chen et al. (2013) originally developed the concept of interval-valued hesitant fuzzy set (IVHFS) in which the membership degree of an element to a given set is not 
precisely defined, but by several possible interval values. Similar to the definition of HFEs, the basic elements of IVHFSs are called interval-valued hesitant fuzzy elements (IVHFEs). For example, suppose two possible interval values, that are $[0.3,0.4]$ and $[0.5,0.6]$, indicating the membership degree of an element, they can be presented by an IVHFE denoted by $\tilde{h}=\{[0.3,0.4],[0.5,0.6]\}$.

Chen et al. (2012) further defined some basic operations of IVHFEs, developed some interval-valued hesitant fuzzy aggregation operators, proposed intervalvalued hesitant fuzzy preference relations to describe hesitant uncertain information in group decision making, and provided numerical examples to show the applications of IVHFSs in the decision making problems. Based on IVHFSs, Bai (2013) developed some distance and similarity measures, Wei and Zhao (2013) developed some induced interval-valued hesitant fuzzy aggregation operators. With interval-valued hesitant fuzzy information, Wei et al. (2014d) investigated multi-criteria decision making problems.

Moreover, Wei (2013) defined a concept called the hesitant interval-valued fuzzy set which is exactly the same as the IVHFS (Chen et al., 2013), and then introduced some aggregation operations of hesitant interval-valued fuzzy sets (Wei et al., 2014d) which are also the same as the interval-valued hesitant fuzzy aggregation operators developed by Chen et al. (2012). Recently, Chen and Xu (2014) derived the properties and relationships of basic operations on IVHFSs, Chen and $\mathrm{Xu}$ (2014) developed some new operations based on Archimedean t-norm and t-conorm.

Qian et al. (2013) developed a concept of generalized hesitant fuzzy set (GHFS) which consists of generalized hesitant fuzzy elements (GHFEs). For example, a decision group consisting of several DMs provides some possible values for the membership degree of an element. Some DMs provide 0.4, some argue between 0.45 and 0.55 , and the others insist on at least 0.6 , then the membership degree can be presented by three intuitionistic fuzzy values (Xu, 2007), which can be denoted by $\alpha_{1}=(0.4,0.6), \quad \alpha_{2}=(0.45,0.45) \quad$ and $\alpha_{3}=(0.6,0)$. So we can get a corresponding GHFE denoted by $h^{g}=(0.4,0.6) \cup(0.45,0.45) \cup(0.6,0)$.

By the envelope operation of intuitionistic fuzzy values, the three intuitionistic fuzzy values can be transformed into three intervals as $\alpha_{1}^{\prime}=[0.4,0.4]$, $\alpha_{2}^{\prime}=[0.45,0.55]$ and $\alpha_{3}^{\prime}=[0.6,1]$, respectively. Thus, $h^{g}$ can also be represented by an IVHFE, denoted by $\tilde{h}^{\prime}=\{[0.4,0.4], \quad[0.45,0.55], \quad[0.6,1]\}$. Furthermore, the basic operations of GHFSs defined by Qian et al. (2013) are consistent with the basic operations of IVHFSs (Chen et al., 2012).

Clearly, IVHFSs and GHFSs are two equivalent extensions of HFSs connected by the envelope operation. The motivation of proposing GHFSs and IVHFSs is to allow the membership degree represented by a margin of error. On the other hand, according to Definition 1, IVHFSs and GFHSs are two special cases of HFSs. All the related operations of IVHFSs and GHFSs are consistent with the corresponding operations of HFSs.

\section{Measures and clustering}

\subsection{Distance and similarity measures}

Distance and similarity measures are important in decision making. Motivated by some famous distance and similarity measures, $\mathrm{Xu}$ and Xia (2011b) originally developed some distance and similarity measures of HFSs, such as the hesitant normalized Hamming distance, the hesitant normalized Euclidean distance, the generalized hesitant normalized distance and the hesitant normalized Hausdorff distance. Moreover, the computation of distance similarity measures require that the HFEs should have the same number of elements. Since the DMs often provided different number of possible values for the membership degree in most cases, Xu and Xia (2011b) proposed an optimistic and a pessimistic rules to extend HFEs to make them have the same length.

According to the generalized hesitant fuzzy weighted distance measure and the generalized hesitant fuzzy ordered weighted distance measure developed by $\mathrm{Xu}$ and Xia (2011b), Peng et al. (2013) further developed a generalized hesitant fuzzy synergetic weighted distance measure, and then investigated some properties and special cases. Recently, Zhang and Xu (2015b) presented a signed distance-based method to compare HFEs by considering a proposed hesitancy index. 


\subsection{Correlation, entropy and cross-entropy measures}

Correlation can reflect a linear relationship between two variables It is an important measure in data analysis, medical diagnosis, pattern recognition, and particularly decision making. Based on an assumption that the HFEs have the same number of elements, and the values in each HFE are arranged in an increasing order, Xu and Xia (2011a) defined some correlation measures on HFEs, Chen et al. (2013) defined some correlation coefficient formulas for HHFs.

$\mathrm{Xu}$ and Xia (2012) defined the entropy and crossentropy measures for hesitant fuzzy information, and then discussed their properties. Farhadinia (2013a) investigated the relationship between the entropy, the similarity measure and the distance measure of HFSs. Farhadinia (2013a) introduced some new formulas for the entropy and the similarity measure of HFSs to provide a transformation from entropy measures to similarity measures.

\subsection{Clustering}

Under hesitant fuzzy environment, Zhang and $\mathrm{Xu}$ (2015c) proposed a clustering algorithm that has advantages in computation based on Boole matrices and similarity measures of HFSs. Zhang and Xu (2015a) proposed a hesitant fuzzy agglomerative hierarchical clustering algorithm for HFSs, and gave some comparisons between this algorithm and the intuitionistic fuzzy hierarchical clustering algorithm proposed by $\mathrm{Xu}$ (2009). Chen et al. (2013) developed some correlation coefficient formulas, and then apply them to clustering analysis. Farhadinia (2013a) developed two clustering algorithms in which new indices of similarity measures for HFSs are applied in data analysis and classification.

\section{Aggregation operators}

In decision making, the aggregation operator is the most used technique to obtain the overall performance of alternatives by aggregating individual preference information. Under intuitionistic fuzzy environment, $\mathrm{Xu}$ (2007); $\mathrm{Xu}$ and Yager (2006) developed some intuitionistic fuzzy aggregation operators. Motivated by these operators, Xia and Xu (2011) developed a series of aggregation operators under hesitant fuzzy environment. Based on quasi-arithmetic means (Hardy et al., 1934), Xia et al. (2013) further defined some quasi-hesitant fuzzy aggregation operators which can be considered as the generations of their previous work (Xia and $\mathrm{Xu}, 2011$ ). Based on these proposed aggregation operators, Liao and $\mathrm{Xu}$ (2014a) defined some hesitant fuzzy hybrid weighted aggregation operators, and then applied them to the multi-criteria decision making problems.

To capture the interrelationship of hesitant fuzzy information, Zhu and $\mathrm{Xu}$ (2013b) developed hesitant fuzzy Bonferroni means, and Zhu et al. (2012b) developed hesitant fuzzy geometric Bonferroni means. In aggregating hesitant fuzzy information, hesitant fuzzy Bonferroni means can consider the whole arguments fairly, while hesitant fuzzy geometric Bonferroni means pay attention to some arguments whose values are too high or too low. Motivated by hesitant fuzzy Bonferroni means, Yu et al. (2012) further defined generalized hesitant fuzzy Bonferroni means and applied them to decision making. Besides Bonferroni means (Bonferroni, 1950), power average operators (Yager, 2001) can also reflect the interrelationship of arguments. Under hesitant fuzzy environment, Zhang (2013) defined a wide range of hesitant fuzzy power average aggregation operators, Liu and Sun (2013) developed some generalized hesitant fuzzy power average aggregation operators.

Considering that the criteria often have different priority levels in multi-criteria decision making, Wei (2012); Yu (2012) developed some hesitant fuzzy prioritized operators. Moreover, Wei et al. (2014c) developed a series of hesitant triangular fuzzy aggregation operators.

\section{Hesitant fuzzy set under linguistic environment}

The advantages of HFSs of handling the imprecise whereby two or more sources of vagueness appearing simultaneously can also be taken into account under the linguistic environment. To improve the modelling and computational abilities of linguistic approaches, Rodriguez et al. (2012) developed the hesitant fuzzy linguistic term set (HFLTS) which enables the DMs hesitate about several possible values to assess a linguistic variable. A context-free grammar is developed to generate rich linguistic expressions that can be represented by means of HFLTs. Basic operations of HFLTSs and their properties are investigated in detail. 
Particularly, they defined the envelope operation of HFLTSs, which is a linguistic interval whose limits are obtained by means of upper bound and lower bound. Actually, the envelop of HFLTSs is the uncertain linguistic variable that was defined by $\mathrm{Xu}$ (2004) in 2004. To show the advantages of HFLTSs in decision making, they also presented a multi-criteria linguistic decision making model, in which the DMs provide their assessments by using linguistic expressions based on comparative terms. Later, to overcome the limitations of the existing linguistic models that use single-valued and predefined terms, Rodríguez et al. (2013) further developed a new linguistic group decision model which is capable of dealing with comparative linguistic expressions based on context-free grammars and HFLTSs. The linguistic group decision model facilitates the elicitation of linguistic information.

To further present linguistic information by HFLTSs, Zhu and Xu (2013a) developed the hesitant fuzzy linguistic preference relation (HFLPR) as a basic decision making tool under hesitant fuzzy linguistic environment. In order to derive meaningful results in decision making by HFLPRs, Zhu and Xu (2013a) developed the consistency measures of HFLPRs to ensure that the DMs are neither being random nor illogical for their preference information, and then established the consistency thresholds of HFLPRs to measure weather a HFLPR is of acceptable consistency. With respect to the inconsistent HFLPRs, two optimization methods were also proposed by Zhu and $\mathrm{Xu}$ (2013a) to improve their consistency until they are acceptable.

Moreover, to compare HFLTSs better, Wei et al. (2014a) gave two possibility degree formulas, and Lee and Chen (2013) developed the concept of likelihoodbased comparison relations. To aggregate hesitant fuzzy linguistic information, Wei et al. (2014a); Zhang and $\mathrm{Wu}$ (2014) proposed some hesitant fuzzy linguistic aggregation operators. For the applications of HFLTSs in decision making, Lin et al. (2014) developed some models for selecting an ERP system.

\section{Applications of HFSs}

To apply HFSs to decision making under uncertainty, Zhu and Xu (2013c) introduced the concept of hesitant fuzzy preference relation (HFPR) to present hesitant fuzzy information, investigated the relationship between HFPRs and fuzzy preference relations (Tanino, 1984), and developed two regression methods to transform HFPRs into fuzzy preference relations. To extend the traditional multiplicative preference relations (Saaty, 1980) (known as comparison matrixes in Analytic Hierarchy Process), Xia and Xu (2013) developed the hesitant multiplicative preference relation (HMPR), and then investigated the group decision making problems.

Under hesitant fuzzy environment, some new decision making methods have been developed. Liao and $\mathrm{Xu}$ (2013) proposed the hesitant fuzzy-VIKOR method based on several developed measures, such as the hesitant normalized Manhattan $L$-metric, the hesitant fuzzy group utility measure, the hesitant fuzzy individual regret measure and the hesitant fuzzy compromise measure. Ma et al. (2014) developed a hesitant fuzzy superiority and inferiority ranking method, and then applied it to deal with the multicriteria group decision making problems.

Based on the presentation of the DMs' preferences in hesitant fuzzy information, Zhang and $\mathrm{Xu}$ (2014) proposed an interval programming method; Zhang and Wei (2013) extended the VIKOR method and the TOPSIS method to solve the group decision making problems; $\mathrm{Xu}$ and Zhang (2013) developed a maximizing deviation method; Wei et al. (2014b) investigated the multi-criteria decision making problems with incomplete weight information; Zhang and Wei (2014) extended the VIKOR method to deal with a correlative multi-criteria decision making problem; Feng et al. (2014) investigated the multi-criteria decision making problems for social network analysis by the TOPSIS method.

$\mathrm{Xu}$ (2014) systematically summarized the theory of HFSs, and showed the applications of HFSs in practice. Recently, Zhu et al. (2015) developed generalized analytical network process (G-ANP) that takes hesitant fuzzy preferences into account. G-ANP eliminates the drawbacks of the fuzzy analytical network process (FANP) (Mikhailov and Singh Madan, 2003) that cannot take consistency into account, and allows multiple forms of preferences, such as crisp (fuzzy) preferences, interval (interval fuzzy) preferences, hesitant (hesitant fuzzy) preferences and stochastic (stochastic fuzzy) preferences. For application, Zhu et al. (2015) applied the G-ANP to a real risk management problem. Moreover, with the idea of hesitancy in decision making, Zhu et al. (2016) developed the hesitant 
analytical hierarchy process and then applied it to evaluate the strategic positions of islands and reefs.

\section{Conclusions}

In this paper, we have reviewed the developments and applications of hesitant fuzzy sets (HFSs). HFSs have the advantage in representing the decision makers' (DMs) preferences characterized by the hesitance whereby two or more possible values can be considered simultaneously for the membership. We have introduced dual hesitant fuzzy sets (DHFSs), extended hesitant fuzzy sets (EHFSs), interval-valued hesitant fuzzy sets (IVHFSs) and generalized hesitant fuzzy sets (GHFSs), and show their relationship with HFSs. Furthermore, we have divided the relevant papers into four groups: the studies on measures and clustering, aggregation operators, HFSs under the linguistic environment, and decision making methods. The HFS is an emerging uncertain decision making tool with numerous studies in recent years. However, how to use HFSs to deal with real-life decision maker problems such as risk decision making, emergency management and big data-driven decision making, and how to apply HFSs to other fields, such as information retrieval and medical diagnosis, may be the highlights for further research.

\section{References}

Krassimir Atanassov. Intuitionistic fuzzy sets. Fuzzy sets and Systems, 20(1) (1986). 87-96.

Zhiyong Bai. Distance similarity measures for interval-valued hesitant fuzzy sets and their application in multicriteria decision making. Journal of Decision Systems, 22(3) (2013). 190-201.

Carlo Bonferroni. Sulle medie multiple di potenze. Bollettino dell'Unione Matematica Italiana, 5(3-4) (1950). 267-270.

$\mathrm{Na}$ Chen, and Z. S. Xu. Properties of interval-valued hesitant fuzzy sets. Journal of Intelligent and Fuzzy Systems, 27(1) (2014). 143-158.

$\mathrm{Na}$ Chen, Z. S. Xu, and M. M. Xia. Interval-valued hesitant preference relations and their applications to group decision making. Knowledge-Based Systems, 37(2012). 528-540

$\mathrm{Na}$ Chen, Z. S. Xu, and M. M. Xia. Correlation coefficients of hesitant fuzzy sets and their applications to clustering analysis. Applied Mathematical Modelling, 37(4) (2013). 2197-2211.

Yuanfang Chen, Xiaodong Peng, Guohua Guan, and Huade Jiang. Approaches to multiple attribute decision making based on the correlation coefficient with dual hesitant fuzzy information. Journal of Intelligent and Fuzzy Systems, 26(5) (2014). 2547-2556.
Didier J Dubois. (1980). Fuzzy sets and systems: theory and applications (Vol. 144): Academic press.

Bahram Farhadinia. Information measures for hesitant fuzzy sets and interval-valued hesitant fuzzy sets. Information Sciences, 240(2013a). 129-144.

Bahram Farhadinia. A novel method of ranking hesitant Fuzzy values for multiple attribute decision-making problems. International Journal of Intelligent Systems, 28(2013b). $752-767$.

Xie Feng, Wanli Zuo, Junhua Wang, and Lizhou Feng. TOPSIS method for hesitant fuzzy multiple attribute decision making. Journal of Intelligent and Fuzzy Systems, 26(5) (2014). 2263-2269.

GH Hardy, JE Littlewood, and G Pólya, Inequalities (1934): Cambridge University Press, Cambridge.

Liwei Lee, and Shyiming Chen. (2013). Fuzzy decision making based on hesitant fuzzy linguistic term sets Intelligent Information and Database Systems (pp. 21-30): Springer.

Huchang Liao, and Zeshui Xu. A VIKOR-based method for hesitant fuzzy multi-criteria decision making. Fuzzy Optimization and Decision Making(2013). 1-20.

Huchang Liao, and Zeshui $\mathrm{Xu}$. Some new hybrid weighted aggregation operators under hesitant fuzzy multi-criteria decision making environment. Journal of Intelligent and Fuzzy Systems, 26(4) (2014a). 1601-1617.

Huchang Liao, and Zeshui Xu. Subtraction and division operations over hesitant fuzzy sets. Journal of Intelligent and Fuzzy Systems, 27(2014b). 65-72.

Rui Lin, Xiaofei Zhao, and Gangxi Wei. Models for selecting an ERP system with hesitant fuzzy linguistic information. Journal of Intelligent and Fuzzy Systems, 26(5) (2014). 2155-2165.

Jing Liu, and Min Sun. Generalized Power Average Operator of Hesitant Fuzzy Numbers and Its Application in Multiple Attribute Decision Making. Journal of Computational Information Systems, 9(8) (2013). 30513058.

Zujun Ma, Nian Zhang, and Ying Dai. A novel SIR method for multiple attributes group decision making problem under hesitant fuzzy environment. Journal of Intelligent and Fuzzy Systems, 26(5) (2014). 2119-2130.

Radko Mesiar, and Andrea Mesiarová-Zemánková. The ordered modular averages. Fuzzy Systems, IEEE Transactions on, 19(1) (2011). 42-50.

Ludmil Mikhailov, and G Singh Madan. Fuzzy analytic network process and its application to the development of decision support systems. Systems, Man, and Cybernetics, Part C: Applications and Reviews, IEEE Transactions on, 33(1) (2003). 33-41.

Dinghong Peng, Chang-Yuan Gao, and Zhi-Fang Gao. Generalized hesitant fuzzy synergetic weighted distance measures and their application to multiple criteria decision-making. Applied Mathematical Modelling, 37(2013). 5837-5850.

Gang Qian, Hai Wang, and Xiangqian Feng. Generalized of Hesitant Fuzzy Sets and their application in decision support system. Knowledge-Based Systems, 37(2013). 357-365.

Rosa M Rodríguez, Luis Martínez, and Francisco Herrera. A group decision making model dealing with comparative 
linguistic expressions based on hesitant fuzzy linguistic term sets. Information Sciences, 241(2013). 28-42.

Rosa M Rodriguez, Luis Martinez, and Francisco Herrera. Hesitant fuzzy linguistic term sets for decision making. Fuzzy Systems, IEEE Transactions on, 20(1) (2012). 109119.

Thoms Saaty. (1980). The analytic hierarchy process: McGraw-Hill, New York.

Tetsuzo Tanino. Fuzzy preference orderings in group decision making. Fuzzy Sets and Systems, 12(2) (1984). 117-131.

Vicenç Torra. Hesitant fuzzy sets. International Journal of Intelligent Systems, 25(6) (2010a). 529-539.

Vicenç Torra. Hesitant fuzzy sets. International Journal of Intelligent Systems, 25(1) (2010b). 529-539.

Rajkumar Verma, and Bhu Dev Sharma. New operations over hesitant fuzzy sets. Fuzzy Information and Engineering, 2(2013). 1-18

Hongjun Wang, Xiaofei Zhao, and Gangwen Wei. Dual hesitant fuzzy aggregation operators in multiple attribute decision making. Journal of Intelligent and Fuzzy Systems, 26(5) (2015). 2281-2290.

Cuiping Wei, Na Zhao, and Xijin Tang. Operators and Comparisons of Hesitant Fuzzy Linguistic Term Sets. Fuzzy Systems, IEEE Transactions on, 22(3) (2014a). 575585.

Guiwu Wei. Hesitant fuzzy prioritized operators and their application to multiple attribute decision making. Knowledge-Based Systems, 31(2012). 176-182.

Guiwu Wei. Some hesitant interval-valued fuzzy aggregation operators and their applications to multiple attribute decision making. Knowledge-Based Systems, 46(2013). 43-53.

Guiwu Wei, Hongjun Wang, Xiaofei Zhao, and Rui Lin. Approaches to hesitant fuzzy multiple attribute decision making with incomplete weight information. Journal of Intelligent and Fuzzy Systems, 26(1) (2014b). 259-266.

Guiwu Wei, Hongjun Wang, Xiaofei Zhao, and Rui Lin. Hesitant triangular fuzzy information aggregation in multiple attribute decision making. Journal of Intelligent and Fuzzy Systems, 41(4) (2014c). 1086-1094.

Guiwu Wei, and Xiaofei Zhao. Induced hesitant intervalvalued fuzzy Einstein aggregation operators and their application to multiple attribute decision making. Journal of Intelligent and Fuzzy Systems, 24(4) (2013). 789-803.

Guiwu Wei, Xiaofei Zhao, Rui Lin, and Hongjun Wang. Models for hesitant interval-valued fuzzy multiple attribute decision making based on the correlation coefficient with incomplete weight information. Journal of Intelligent and Fuzzy Systems, 26(4) (2014d). 1631-1644.

Meimei Xia, and Zeshui Xu. Hesitant fuzzy information aggregation in decision making. International Journal of Approximate Reasoning, 52(3) (2011). 395-407.

Meimei Xia, and Zeshui Xu. Managing hesitant information in GDM problems under fuzzy and multiplicative preference relations. International Journal of Uncertainty, Fuzziness and Knowledge-Based Systems, 21(06) (2013). 865-897.

Meimei Xia, Zeshui Xu, and $\mathrm{Na}$ Chen. Some hesitant fuzzy aggregation operators with their application in group decision making. Group Decision and Negotiation, 22(2) (2013). 259-279.
Zeshui $\mathrm{Xu}$, and Meimei Xia. On distance and correlation measures of hesitant fuzzy information. International Journal of Intelligent Systems, 26(5) (2011a). 410-425.

Zeshui Xu. Uncertain linguistic aggregation operators based approach to multiple attribute group decision making under uncertain linguistic environment. Information Sciences, 168(1) (2004). 171-184.

Zeshui Xu. Intuitionistic fuzzy aggregation operators. Fuzzy Systems, IEEE Transactions on, 15(6) (2007). 1179-1187.

Zeshui $\mathrm{Xu}$. Intuitionistic fuzzy hierarchical clustering algorithms. Journal of Systems Engineering and Electronics, 20(1) (2009). 90-97.

Zeshui Xu. (2014). Hesitant fuzzy sets theory (Vol. 314) Springer.

Zeshui Xu, and Meimei Xia. Distance and similarity measures for hesitant fuzzy sets. Information Sciences, 181(11) (2011b). 2128-2138

Zeshui Xu, and Meimei Xia. Hesitant fuzzy entropy and crossentropy and their use in multiattribute decision-making. International Journal of Intelligent Systems, 27(9) (2012). 799-822.

Zeshui $\mathrm{Xu}$, and Ronald $\mathrm{R}$ Yager. Some geometric aggregation operators based on intuitionistic fuzzy sets. International journal of general systems, 35(4) (2006). 417-433.

Zeshui $\mathrm{Xu}$, and Xiaolu Zhang. Hesitant fuzzy multi-attribute decision making based on TOPSIS with incomplete weight information. Knowledge-Based Systems, 52(2013). 53-64.

Ronald R Yager. On the theory of bags. International Journal of General System, 13(1) (1986). 23-37.

Ronald R Yager. On ordered weighted averaging aggregation operators in multicriteria decisionmaking. Systems, Man and Cybernetics, IEEE Transactions on, 18(1) (1988). 183-190.

Ronald R Yager. Families of OWA operators. Fuzzy sets and systems, 59(2) (1993). 125-148.

Ronald R Yager. The power average operator. Systems, Man and Cybernetics, Part A: Systems and Humans, IEEE Transactions on, 31(6) (2001). 724-731.

Jun Ye. Correlation coefficient of dual hesitant fuzzy sets and its application to multiple attribute decision making. Applied Mathematical Modelling, 38(2) (2013). 659-666.

Dejian Yu. Hesitant fuzzy prioritized operators and their application in multi-criteria group decision making. African Journal of Business Management, 6(33) (2012). 9370-9380.

Dejian $\mathrm{Yu}$, Yingyu $\mathrm{Wu}$, and Wei Zhou. Generalized hesitant fuzzy Bonferroni mean and its application in multi-criteria group decision making. Journal of information \& Computational Science, 9(2) (2012). 267-274.

Lotfi A. Zadeh. Fuzzy sets. Information and Control 8(1965). 338-353.

Lotfi A. Zadeh. The concept of a linguistic variable and its application to approximate reasoning-I. Information sciences, 8(3) (1975). 199-249.

Nian Zhang, and Guiwu Wei. Extension of VIKOR method for decision making problem based on hesitant fuzzy set. Applied Mathematical Modelling, 37(2013). 4938-4947.

Nian Zhang, and Guiwu Wei. A multiple criteria hesitant fuzzy decision making with Shapley value-based VIKOR method. Journal of Intelligent and Fuzzy Systems, 26(2) (2014). 1065-1075. 
Xiaolu Zhang, and Z.S. Xu. Hesitant fuzzy agglomerative hierarchical clustering algorithms. International Journal of Systems Science, 46(3) (2015a). 562-576.

Xiaolu Zhang, and Zeshui Xu. Interval programming method for hesitant fuzzy multi-attribute group decision making with incomplete preference over alternatives. Computers \& Industrial Engineering, 75(2014). 217-229.

Xiaolu Zhang, and Zeshui Xu. Hesitant fuzzy QUALIFLEX approach with a signed distance-based comparison method for multiple criteria decision analysis. Expert Systems with Applications, 42(2) (2015b). 873-884.

Xiaolu Zhang, and Zeshui Xu. Novel distance and similarity measures on hesitant fuzzy sets with applications to clustering analysis. Journal of Intelligent \& Fuzzy Systems, 28(5) (2015c). 2279-2296.

Zhiming Zhang. Hesitant fuzzy power aggregation operators and their application to multiple attribute group decision making. Information Sciences, 234(2013). 150-181.

Zhiming Zhang, and Chong Wu. Hesitant fuzzy linguistic aggregation operators and their applications to multiple attribute group decision making. Journal of Intelligent and Fuzzy Systems, 26(5) (2014). 2185-2202.

Bin Zhu, and Z. S. Xu. Consistency measures for hesitant fuzzy linguistic preference relations. Fuzzy Systems, IEEE Transactions on, 22(1) (2013a). 35-45.
Bin Zhu, and Z. S. Xu. Hesitant fuzzy Bonferroni means for multi-criteria decision making. Journal of the Operational Research Society, 64(12) (2013b). 1831-1840.

Bin Zhu, and Zeshui Xu. Regression methods for hesitant fuzzy preference relations. Technological and Economic Development of Economy, 19(sup1) (2013c). S214-S227.

$\mathrm{Bin} \mathrm{Zhu}$, and Zeshui Xu. Some results for dual hesitant fuzzy sets. Journal of Intelligent and Fuzzy Systems, 26(2014). 1657-1668.

Bin Zhu, and Zeshui Xu. Extended hesitant fuzzy sets Technological and Economic Development of Economy, 22(1) (2016). 100-121.

Bin Zhu, Zeshui Xu, and Meimei Xia. Dual Hesitant Fuzzy Sets. Journal of Applied Mathematics, 2012(2012a). 1-13.

Bin Zhu, Zeshui Xu, and Meimei Xia. Hesitant fuzzy geometric Bonferroni means. Information Sciences, 205(2012b). 72-85

Bin Zhu, Zeshui Xu, Ren Zhang, and Mei Hong. Generalized analytic network process. European Journal of Operational Research, 244(1) (2015). 277-288.

Bin Zhu, Zeshui Xu, Ren Zhang, and Mei Hong. Hesitant analytic hierarchy process. European Journal of Operational Research, 250(2) (2016). 602-614.

Hans Jürgen Zimmermann. (2001). Fuzzy set theory-and its applications: Springer. 